\title{
The copper-dependent ACE1 transcription factor activates the transcription of the mco 1 gene from the basidiomycete Phanerochaete chrysosporium
}

Correspondence

Rafael Vicuña

rvicuna@bio.puc.cl

Received 12 September 2007

Revised 31 October 2007

Accepted 7 November 2007

\author{
Paulo Canessa,† José Miguel Álvarez,† Rubén Polanco,ł \\ Paulina Bull and Rafael Vicuña
}

\begin{abstract}
Departamento de Genética Molecular y Microbiología, Facultad de Ciencias Biológicas, Pontificia Universidad Católica de Chile and Instituto Milenio de Biología Fundamental y Aplicada, Santiago, Chile
\end{abstract}

\begin{abstract}
We have previously identified and functionally characterized the transcription factor ACE1 (Pc-ACE1) from Phanerochaete chrysosporium. In Saccharomyces cerevisiae, ACE1 activates the copper-dependent transcription of target genes through a DNA sequence element named ACE. However, the possible target gene(s) of Pc-ACE1 were unknown. An in silico search led to the identification of putative ACE elements in the promoter region of $m c 01$, one of the four clustered genes encoding multicopper oxidases (MCOs) in P. chrysosporium. Since copper exerts an effect at the transcriptional level in MCOs from several organisms, in this work we analysed the effect of copper on transcript levels of the clustered MCO genes from $P$. chrysosporium, with the exception of the transcriptionally inactive mco3. Copper supplementation of cultures produced an increment in transcripts from genes mco1 and mco2, but not from mco4. Electrophoretic mobility-shift assays revealed that Pc-ACE1 binds specifically to a probe containing one of the putative ACE elements found in the promoter of mco1. In addition, using a cell-free transcription system, we showed that in the presence of cuprous ion, PC-ACE1 induces activation of the promoter of $m c 01$, but not that of $m c 02$.
\end{abstract}

\section{INTRODUCTION}

The lignin-degrading basidiomycete Phanerochaete chrysosporium has an impressive array of extracellular enzymes involved in lignocellulose degradation. Among them, the extensively characterized lignin and manganese peroxidases (LiP and $\mathrm{MnP}$, respectively) have been described as true ligninases because of their high redox potential (Martinez et al., 2005; Kersten \& Cullen, 2007). A third kind of enzyme also involved in lignin degradation, although not produced by $P$. chrysosporium, is the phenol oxidase termed laccase (Larrondo et al., 2003; Baldrian, 2006). Laccases belong to the family of multicopper oxidases (MCOs), a large group of copper-containing proteins that include mammalian ceruloplasmin, plant ascorbate oxidases and fungal Fet3 ferroxidases (Solomon et al., 1996). Spectroscopic and X-ray crystallographic studies have revealed that MCOs contain at least one blue copper or

†These authors contributed equally to this work.

łPresent address: Depto de Ciencias Biológicas, Facultad de Ciencias de la Salud, Universidad Andrés Bello, Av. República 217, Santiago, Chile.

Abbreviations: EMSA, electrophoretic mobility-shift assay; MCO, multicopper oxidase; qRT-PCR, real-time quantitative RT-PCR.

A supplementary figure is available with the online version of this paper. type 1 site (T1) and a type 2/type 3 (T2/T3) trinuclear copper cluster as the minimal functional unit. These copper centres, located in the active site of these enzymes, play a key role in catalysis (Solomon et al., 1996; Baldrian, 2006).

In addition to the structural and catalytic roles played by copper, some reports have shown that this metal also affects the expression of some MCOs. For instance, copper increases mammalian ceruloplasmin mRNA levels in hepatoma cells through copper-induced promoter activation (Martin et al., 2005). Copper has also been shown to activate the transcription of CmAO4, a gene encoding ascorbate oxidase in the plant Cucumis melo (Sanmartin et al., 2007), as well as to increase mRNA levels of fet3, a gene encoding a ferroxidase involved in iron uptake in Saccharomyces cerevisiae (Gross et al., 2000). Laccases are not an exception in this respect. Thus, copper regulates laccase mRNA levels in the fungi Trametes versicolor (Collins \& Dobson, 1997), Ceriporiopsis subvermispora (Karahanian et al., 1998), Pleurotus ostreatus (Palmieri et al., 2000), Pleurotus sajor-caju (Soden \& Dobson, 2001, 2003) and Trametes pubescens (Galhaup et al., 2002).

Analysis of the promoter region of some of the MCOencoding genes has shown the presence of a copperdependent responsive element named ACE (activation of 
cup1 expression), that was previously described in yeast as the recognition site for the ACE1 transcription factor (Evans et al., 1990). ACE1 is a member of a group of fungal transcription factors that contain a copper-fist cysteine-rich DNA-binding domain located in the $\mathrm{N}$-terminal region $(\mathrm{Hu}$ et al., 1990). This trans-acting regulatory protein activates the transcription of the $S$. cerevisiae metallothioneinencoding genes cup1 (Thiele, 1988; Buchman et al., 1989; Culotta et al., 1989; Evans et al., 1990; Thorvaldsen et al., 1993) and crs5 (Culotta et al., 1994) and the superoxide dismutase gene (sod1) (Gralla et al., 1991; Carri et al., 1991) in response to copper. This metal is essential for the DNAbinding activity of ACE1 (Winge, 1998). Interestingly, we have recently identified the gene encoding ACE1 in $P$. chrysosporium (Pc-ace1), the first basidiomycetal orthologue of the yeast ACE1 transcription factor (Polanco et al., 2006). However, until this work, the possible target gene(s) of PcACE1 remained unknown.

Previously, we have shown that the genome database of $P$. chrysosporium (http://genome.jgi-psf.org/Phchr1/Phchr1. home.html) lacks laccase-encoding sequences. Instead, we identified four clustered MCO genes (designated mcol to mco4) distantly related to laccases (Larrondo et al., 2003, 2004). Heterologous expression of mco1-cDNA in Aspergillus nidulans showed that the substrate specificity of the recombinant MCO1 differs from that of laccases. For example, MCO1 has strong ferroxidase activity, with a $K_{\mathrm{m}}$ value similar to Fet3 protein from S. cerevisiae (Larrondo et al., 2003). Although extracellular MCO1 shows biochemical and structural similarities with the membrane-bound Fet3 from yeast, we have recently identified and characterized $P c$-fet 3 , the gene encoding the canonical ferroxidase involved in iron uptake in P. chrysosporium (Larrondo et al., 2007a). The presence of Pc-fet 3 and the lack of a C-terminal transmembrane domain anchor in MCO1, which is distinctive of Fet3 proteins, supports the assertion that MCO1 and Fet3 play different roles in the cell (Larrondo et al., 2003, 2007a). A recent and detailed phylogenetic analysis of more than $350 \mathrm{MCOs}$, including those from $P$. chrysosporium, supports this statement (Hoegger et al., 2006). To date, the physiological function of MCO1 remains obscure.

In an effort to gain insight into the role of MCO1, we decided to analyse the transcriptional regulation of $\mathrm{mcol}$ and the molecular mechanisms involved in it. The fact that copper affects the transcription of various $\mathrm{MCO}$-encoding genes in different organisms, plus the presence of putative ACE elements in the promoter of mcol (Polanco et al., 2006), led us to analyse the effect of copper on the expression of this novel group of multicopper oxidases recently discovered in P. chrysosporium (Larrondo et al., 2003). In this work we show that $m c o 1$ is a target gene of the recently described ACE1 transcription factor in P. chrysosporium.

\section{METHODS}

Strain and culture conditions. P. chrysosporium homokaryotic strain RP-78 was obtained from the Center for Mycology Research,
Forest Products Laboratory, Madison, WI, USA. For solid-state incubations, $P$. chrysosporium spores were inoculated on potatodextrose agar plates and grown for 1 week at $39{ }^{\circ} \mathrm{C}$. Spores were collected by flooding the agar plates with $5 \mathrm{ml}$ sterile water, and $2 \times 10^{6}$ spores were inoculated in $20 \mathrm{ml}$ carbon-limited stationary liquid cultures as previously described (Brown et al., 1993). Cultures were harvested after 4 days of incubation. When indicated, they were supplemented with $\mathrm{CuSO}_{4}$ to a final concentration of $0.25 \mathrm{mM}$ for the indicated times.

Analysis of the promoter regions of mco genes. Using the reported cDNA sequences of $m c o 1, m c o 2, m c o 3$ and $m c 04$ (GenBank accession numbers AY225437, AY532139, AY532142 and AY532149, respectively) and by means of BLAST, $m c o$ genes were localized in the $P$. chrysosporium genome database (http://genome.jgi-psf.org/Phchrl/ Phchr1.home.html). Taking into consideration the presence of several gene models, each promoter was manually obtained and arbitrarily defined as the corresponding intergenic region. This procedure led to uncovering of promoter regions of 1407, 827 and 537 bp for mco 1, mco 2 and $m c 03$, respectively. Since the closest upstream gene model for $m c 04$ is located $5.2 \mathrm{kbp}$ away, the promoter region was arbitrarily defined as the sequence $1500 \mathrm{bp}$ upstream from the mco4 translation start site. These sequences were examined for the presence of putative ACE elements using the MatInspector software (http://www.genomatix. de/products/MatInspector/). The analysis was restricted to fungal transcription binding sites.

RNA extraction. After 4 days of growth, the mycelia obtained from five independent flasks (processed as a batch) were separated from the culture fluid by filtration through Miracloth (Calbiochem) and frozen in liquid nitrogen. The frozen mycelium was ground to a powder in a mortar containing liquid nitrogen. The powder was homogenized in the same mortar by the addition of $4 \mathrm{ml}$ Tris/ $\mathrm{HCl}$ buffer $(0.2 \mathrm{M}$ Tris/ $\mathrm{HCl} \mathrm{pH} 7.5,0.5 \mathrm{M} \mathrm{NaCl}, 0.01 \mathrm{M}$ EDTA, $1 \%$ SDS, $50 \mathrm{mM} \beta$ mercaptoethanol) and $4 \mathrm{ml}$ phenol/chloroform/isoamyl alcohol ( $25: 24: 1$, by vol.). Each sample was transferred to a $50 \mathrm{ml}$ RNasefree tube and vortex-homogenized. In the same tube and using a sterile syringe and needle, the fluid was pipetted up and down several times to shear genomic DNA (gDNA). Thereafter, the fluid was transferred to four $2 \mathrm{ml}$ Eppendorf tubes. After centrifugation (15 min at 14000 r.p.m.), the aqueous phase was phenol-extracted in a clean tube and the RNA was obtained as described by Manubens et al. (2003). Ten micrograms of total RNA was fractionated by electrophoresis in a formaldehyde-agarose gel $(1.2 \% \mathrm{w} / \mathrm{v})$ and RNA integrity was verified by ethidium-bromide staining. Poly(A) mRNA was obtained from $100 \mu \mathrm{g}$ total RNA using the mRNA DIRECT kit (Dynal), according to manufacturer's instructions. To ensure the absence of gDNA, the poly(A) mRNA obtained was incubated at $37{ }^{\circ} \mathrm{C}$ for 30 min using RQ1 RNase-free DNase (Promega), according to the manufacturer's instructions. For each sample, $2 \mu \mathrm{l}$ treated mRNA was collected before proceeding with the retrotranscription step. To check for potential gDNA contamination, RT-minus reactions were carried out in real time using SYBR Green detection chemistry. No cycle threshold $\left(C_{\mathrm{t}}\right)$ values were obtained, at least under the cycle conditions used (see below). Only those mRNA samples that did not show gDNA contamination were reverse transcribed using the MMLV reverse transcriptase (Invitrogen) for $45 \mathrm{~min}$ at $42{ }^{\circ} \mathrm{C}$, according to manufacturer's instructions. The cDNA obtained was diluted twofold.

Real-time quantitative RT-PCR (qRT-PCR). Relative quantification of mco transcript levels was performed in real time using the Brilliant SYBR Green QPCR Master Reagent kit and the Mx3000P detection system (Stratagene). Primer sequences and predicted $T_{\mathrm{m}}$ values, as well as amplicon length, are shown in Table 1 . Levels of mRNA from the glyceraldehyde-3-phosphate dehydrogenase ( $g a p d h$ ) gene were used for normalization. The qRT-PCR mixture $(25 \mu \mathrm{l})$ 
Table 1. Oligonucleotides employed for qRT-PCR

The $T_{\mathrm{m}}$ values were calculated using the nearest-neighbour method (Breslauer et al., 1986).

\begin{tabular}{|llcc|}
\hline Primer & Sequence $\left(\mathbf{5}^{\prime} \mathbf{3}^{\prime}\right)$ & Predicted $\boldsymbol{T}_{\mathbf{m}}\left({ }^{\circ} \mathbf{C}\right)$ & Amplicon length $(\mathbf{b p})$ \\
\hline mco $1 \mathrm{Fw}$ & CATAACCGAGTGCGGAATACC & 58.6 & 101 \\
mco $1 \mathrm{Rv}$ & GCGTGTCATAATGCGAATGC & 59.3 & 107 \\
mco2 $\mathrm{Fw}$ & CTCCGGTCAAATTCCCTAGCT & 58.9 & \\
mco2 $\mathrm{Rv}$ & ACCCCAGGGCGAAAGGTA & 59.5 & \multirow{2}{*}{81} \\
mco $4 \mathrm{Fw}$ & TGCGATAAACGGTATTGGTCAGT & 59.9 & \\
mco $4 \mathrm{Rv}$ & TATAAGACGCAGGCGGTAGGT & 58.1 & \\
gapdh $\mathrm{Fw}$ & TGCTGATGCCCCCATGTT & 60.0 & \\
gapdh $\mathrm{Rv}$ & CAGGACGCGTTCGAGATG & 58.0 & \\
\hline
\end{tabular}

contained $2.0 \mu \mathrm{l} \mathrm{cDNA}$ template and $140 \mathrm{nM}$ of each primer. The qRT-PCR was performed under the following conditions: $10 \mathrm{~min}$ at $95{ }^{\circ} \mathrm{C}$ followed by 40 cycles of $30 \mathrm{~s}$ at $94{ }^{\circ} \mathrm{C}, 30 \mathrm{~s}$ at $56{ }^{\circ} \mathrm{C}$ and $30 \mathrm{~s}$ at $72{ }^{\circ} \mathrm{C}$, followed by a melting cycle from $55{ }^{\circ} \mathrm{C}$ to $95{ }^{\circ} \mathrm{C}$ to check for amplification specificity. $C_{\mathrm{t}}$ values were obtained during the elongation period of the qRT-PCR. A previous standard quantification curve with several serial dilutions of RT-PCR products was constructed for each gene to calculate the amplification efficiency (E) according to the equation $E=\left[10^{(-1 / \text { slope })}\right]-1$. The $E$ values obtained for mcol, mco2, mco4 and gapdh were $96 \%$, 99\%, $98 \%$ and $94 \%$, respectively. These values were used to obtain a more accurate ratio between the gene of interest (GOI) and the expression of the housekeeping (HK) gene, using the following equation: $\left[\left(1+E_{\mathrm{GOI}}\right)^{-[\mathrm{CtGOI}-\mathrm{CtGOI} \text { control }]}\right] /\left[\left(1+\mathrm{E}_{\mathrm{HK}}\right)^{-[\mathrm{CtHK}-\mathrm{CtHK} \text { control }]}\right]$. Values are referred to the culture not supplemented with copper (control). All experiments were performed in two biological and three technical replicates.

\section{In vitro transcription-translation of the $P$. chrysosporium} ACE1 transcription factor (Pc-ACE1). The previously cloned PcACE1 transcription factor (Polanco et al., 2006) was subcloned into the NdeI and BamHI sites of the bacterial expression vector pET21a (Novagen). Primers to obtain the S. cerevisiae ACE1 (Sc-ACE1) coding sequence (5'-CATATGGTCGTAATTAACGGGGTCA-3', direct, and 5'-GGATCCCCTTGTGAATGTGAGTTA-3', reverse) were designed in accordance with the reported sequence (GenBank accession number AY557820). Because of the absence of introns, the Sc-ACE1 coding sequence was obtained by PCR using gDNA. The ScACE1 transcription factor was also cloned into the NdeI and BamHI sites of the pET21a vector. Nucleotide sequences were determined with the ABI Prism Big Dye terminator cycle sequencing kit on ABI automated sequencers (Applied Biosystems). Bacterial heterologous expression was unsuccessful (data not shown). Therefore, taking a different approach, both plasmids were linearized at the BamHI site and used for in vitro synthesis of their corresponding mRNA using the Riboprobe in vitro Transcription System (Promega). mRNA integrity and size specificity was verified by ethidium-bromide staining (see supplementary Fig. S1, available with the online version of this paper). One microgram of each mRNA was further incubated for $90 \mathrm{~min}$ at $30{ }^{\circ} \mathrm{C}$ with the Flexi Rabbit Reticulocyte Lysate System (Promega) in the presence of $\left[{ }^{35} \mathrm{~S}\right]$ methionine. Thereafter, $1 \mu \mathrm{l}$ of each translation product was separated by SDS-PAGE using standard procedures. The SDS-PAGE gel was fixed, dried and exposed on scientific autoradiographic imaging films (Kodak), thus allowing the confirmation of the predicted molecular mass of each protein (see Fig. S1).

Preparation of labelled probes. A probe containing two previously described ACE elements from the S. cerevisiae cup1 gene (Culotta et al., 1989) was PCR-amplified using primers 5'-GATTCTTTTGCTGGCATTTC-3' (direct) and 5' -GACAATCCATATTGCGTTG-3' (reverse). A specific PCR product was obtained, cloned into pGEM$\mathrm{T}$ vector (Promega) and sequenced. The probe was PCR-amplified in the presence of $\left[\alpha_{-}{ }^{32} \mathrm{P}\right] \mathrm{dCTP}$ using the obtained plasmid as a template. In addition, ${ }^{32} \mathrm{P}$-end-labelled double-stranded oligomers containing one of the ACE elements identified in the promoter region of mcol were used as probe. Radioactive labelled probes were purified by PAGE.

Electrophoretic mobility-shift assays (EMSAs). Standard binding reaction mixtures $(30 \mu \mathrm{l})$ contained: $30 \mathrm{mM}$ HEPES/KOH buffer pH 7.5, $2.0 \mathrm{mM}$ EDTA, $0.1 \mathrm{mM} \mathrm{ZnSO}, 0.12 \mathrm{mM} \mathrm{AgNO} 3,40 \mathrm{mM}$ $\mathrm{KCl}, 7.5 \mathrm{mM}$ DTT, $12 \%$ glycerol, $3 \%$ PEG 4000, $30 \mathrm{ng}$ poly(dI-dC) and $300 \mathrm{ng}$ Bluescript KSII plasmid. The concentrations of $\mathrm{CuSO}_{4}$ and ascorbic acid (included to reduce $\mathrm{Cu}^{2+}$ to $\mathrm{Cu}^{+}$) were $0.15 \mathrm{mM}$, unless otherwise specified. To each binding reaction, $2 \mu \mathrm{l}$ rabbit reticulocyte lysate containing the synthesized Sc-ACE1 or Pc-ACE1 transcription factor was added, as well as $50 \mathrm{ng}$ (20000-40000 c.p.m.) labelled DNA probe. After incubation for $15 \mathrm{~min}$ at room temperature, samples were separated by electrophoresis as described previously (Polanco et al., 2002). No DNA-protein interaction was observed when binding assays were carried out with $2 \mu \mathrm{l}$ rabbit reticulocyte lysate in which the translation reaction was omitted (data not shown). Binding assays shown in Fig. 3(D) were carried out in the absence of $\mathrm{AgNO}_{3}$. For competition experiments, labelled probe was premixed with the unlabelled probe or the non-specific competitor.

Pc-ACE1-mediated in vitro transcription. Promoter activation mediated by Pc-ACE1 was studied using an in vitro transcription system. First, a 500 bp DNA fragment corresponding to the upstream sequence from the mcol translation start site containing one ACE element was PCR amplified using primers $5^{\prime}$-ATTAATCTAGAGGCGCTCCCAGACC-3' (direct) and 5'-AAGCTTGCGATAATGCAAAGTGAAGGATG-3' (reverse). After this procedure, the $589 \mathrm{bp}$ CMV promoter of the mammalian expression vector pEGFP-N1 (Clontech) was removed using AseI and HindIII restriction endonucleases and replaced with the aforementioned $500 \mathrm{bp} \mathrm{mcol}$ promoter fragment. Similarly, the complete $\mathrm{mco} 2$ promoter (as defined above, 'Analysis of the promoter regions of mco genes'; $827 \mathrm{bp)}$ was PCR-amplified using primers 5'-ATTAATGGCGAGGGTGCCGTTGCT-3' (direct) and 5'-AAGCTTTGCGAGCAGAGGCGGTGG-3' (reverse) and cloned into the pEGFP-N1 vector as mentioned. In each case, a plasmid containing the respective mco promoter region was linked to an EGFP reporter gene. In vitro transcription mixtures $(25 \mu \mathrm{l})$ contained $3.3 \mu \mathrm{l} \mathrm{HeLa}$ nuclear extract (Promega), $0.4 \mathrm{mM}$ of each ribonucleotide, $100 \mathrm{ng}$ linearized plasmid DNA template, 40 units RNase OUT and $3 \mu \mathrm{l}$ rabbit reticulocyte lysate containing the in vitro-synthesized Pc-ACE1 transcription factor in $20 \mathrm{mM}$ HEPES $\mathrm{pH} 7.9,100 \mathrm{mM} \mathrm{KCl}, 0.2 \mathrm{mM}$ EDTA, $0.5 \mathrm{mM}$ DTT, $3 \mathrm{mM} \mathrm{MgCl}, 20 \%$ (v/v) glycerol. When indicated, reaction mixtures were also supplemented with $25 \mu \mathrm{M} \mathrm{CuSO}_{4}, 25 \mu \mathrm{M}$ 
ascorbate and $25 \mu \mathrm{M} \mathrm{KCN}$. Control reactions were carried out with $3 \mu \mathrm{l}$ rabbit reticulocyte lysate in which the translation reaction was omitted. Following transcription, reporter gene mRNA levels were measured by qRT-PCR using primers 5'-GAGAAGCGCGATCACATGGT-3' (direct) and 5'-TTACTTGTACAGCTCGTCCATGC$3^{\prime}$ (reverse), as described above.

\section{RESULTS}

\section{Effect of copper on transcript levels of mco genes}

The effect that copper exerts at the transcriptional level of $m c o$ genes in various organisms (see above), and the previous detection of putative ACE elements in the promoter of $m c o 1$ from $P$. chrysosporium (Polanco et al., 2006), prompted us to examine whether this metal regulates the expression of mcol, mco2 and mco4. As described previously, the $m c 03$ gene is transcriptionally inactive due to the insertion of an $8.14 \mathrm{~kb}$ gypsy-class retroelement within the 12th intron (Larrondo et al., 2007b). Therefore, it was not analysed in this study. As shown in Fig. 1, the addition of copper to the culture medium led to a 4-fold increase in mcol-transcript levels after $30 \mathrm{~min}$, while $m c 02$ transcripts were increased 2.5fold. In the case of mco4, no major change was observed during the period analysed.

\section{Analysis of $\boldsymbol{m c o}$ promoter regions}

These results prompted us to examine the promoters of mco genes to look for the presence of ACE elements. The

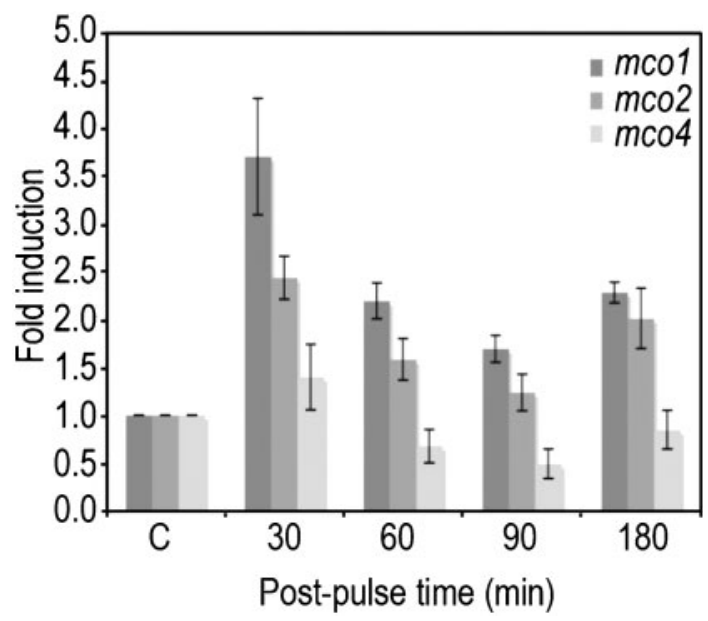

Fig. 1. Effect of $\mathrm{Cu}^{2+}$ on transcript levels from mco genes. $P$. chrysosporium was grown for 4 days in $\mathrm{C}$-starved medium and then harvested ( $\mathrm{C}$, Control) or exposed to $\mathrm{CuSO}_{4}$ to a final concentration of $250 \mu \mathrm{M}$ for the indicated times. After each period of time, the cultures were harvested, mRNA was isolated and qRTPCR was performed as described in Methods. The mco3 gene was not analysed since it is transcriptionally inactive (Larrondo et al., 2007b). four clustered mco genes (mcol to mco4) are located on Scaffold 9 of the assembly version 2.0 of the $P$. chrysosporium genome database (Larrondo et al., 2004). Their respective promoter regions were defined and searched for the presence of regulatory elements as described in Methods. As expected, two putative ACE elements were identified in the promoter of mcol (Polanco et al., 2006) (Fig. 2A). Both adhere to the consensus sequence of the previously described ACE elements in the promoter regions of the $S$. cerevisiae cup1 and sod 1 genes (Fig. 2B). The ACE sequences in the promoter of mcol are centred between positions -306 to -292 and -849 to -835 from the $m c o 1$ translation start site (Fig. 2C). On the other hand, although mco2-transcript levels showed a slight increase (Fig. 1), no putative ACE elements were identified in its promoter. Likewise, no ACE-like sequences were found in the upstream regulatory region of mco4.

\section{DNA-binding activity of Pc-ACE1}

It seemed highly likely that the higher levels of $\mathrm{mcol}$ transcripts observed upon incubation of cultures with copper are mediated by Pc-ACE1. In order to test this hypothesis, as a first approach we characterized the DNAbinding activity of the in vitro-synthesized Pc-ACE1 protein to the two previously described ACE elements from the S. cerevisiae cup1 gene (Culotta et al., 1989). The EMSAs shown in Fig. 3 illustrate the specific binding of PcACE1 to a DNA probe containing these sequences. In this experiment, two DNA-protein complexes were observed (Fig. 3A, lane 2). The lower-mobility complex appears as a slight band signal that shows some level of competition with the unlabelled probe. On the other hand, a stronger band corresponding to a higher-mobility DNA-protein complex was readily displaced when binding assays were carried out in the presence of a molar excess of unlabelled DNA probe (Fig. 3A, lanes 3-5). Displacement was much more limited when assays were conducted in the presence of non-specific DNA competitor (Fig. 3A, lanes 6-8). Similar results were obtained using the same DNA probe with the in vitro-synthesized ACE1 protein from $S$. cerevisiae, used as a positive control (see supplementary Fig. S1, available with the online version of this paper).

In order to determine whether Pc-ACE1 can specifically interact with a probe containing one of the ACE sequences identified in the promoter of $m c o l$, a probe containing the element located between positions -306 and -292 was synthesized (Fig. 3B). As shown in Fig. 3(C), this ${ }^{32} \mathrm{P}-$ labelled 40 bp oligomer forms an easily observed DNAprotein complex with Pc-ACE1. An equivalent result was also observed using a $100 \mathrm{bp}$ DNA probe (data not shown). To confirm the specificity of this association, competition experiments were carried out using double-stranded unlabelled 40 bp DNA oligomers containing either the same ACE element or a mutant version of this ACE with five point mutations (Fig. 3B). Formation of the DNAprotein complex was completely inhibited by incubation 


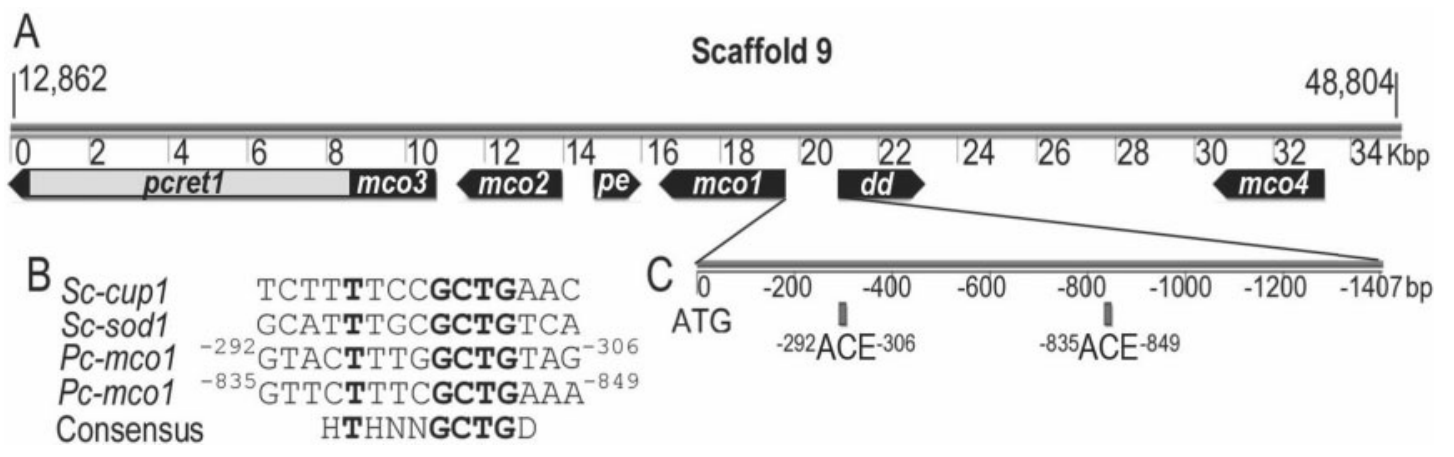

Fig. 2. Representation of the four clustered mco genes from $P$. chrysosporium. (A) The schematic representation (to scale) shows the four mco genes and some neighbouring gene models (pe, phosphoesterase; $d d$, dihydrolipoamide dehydrogenase) located on Scaffold 9, between coordinates 12862 and 48804 . To simplify the figure, two gene models between the dihydrolipoamide dehydrogenase gene model and mco 4 were omitted. Genes and gene models are represented in black boxes showing transcriptional orientation, whereas promoters and intergenic regions are shown in white spaces. The $8.14 \mathrm{kbp} p c r e t 1$ retroelement (grey box) interrupts the mco3 gene (Larrondo et al., 2007b). mco1 promoter, 1407 bp; mco2 promoter, 827 bp; mco3 promoter, $537 \mathrm{bp}$; mco4 promoter, $1500 \mathrm{bp}$. (B) Alignment of the ACE elements from the S. cerevisiae cup1 and sod1 genes (Sc-cup1 and Sc-sod1) and the two ACE elements identified in the promoter of mco1. All ACE elements adhere to the consensus sequence $H(T) H N N G C T G D(H$, not $G, N$, any nucleotide, $D$, not $C)$. Residues shown in bold type have been assigned a key role involved in DNA-protein interaction (Gralla et al., 1991; Beaudoin et al., 2003). (C) The mco1 promoter region, as defined in Methods. ACE elements (grey boxes) are denoted with their respective positions (superscript numbers) relative to the mco1 translation start site (ATG).

with excess wild-type oligomer, whereas the mutant competitor was less effective (Fig. 3C). Although this result indicates that the mutated sequence is important for the observed protein-DNA interaction, some additional DNA sequences may also be involved in complex stabilization.

A further control of binding specificity was conducted by measuring the effect of copper on complex formation. As indicated in Methods, standard binding assays were conducted in the presence of this metal. No DNA-protein complex was observed in the absence of copper (Fig. 3D, lane 7). Addition of $\mathrm{KCN}$, a specific chelator of $\mathrm{Cu}^{+}$, inhibited the DNA-protein complex formation in a concentration-dependent manner (Fig. 3D, lanes 3-5). To avoid copper reduction, a binding assay containing $25 \mu \mathrm{M}$ $\mathrm{CuSO}_{4}$ was also carried out, in the absence of ascorbic acid. As shown in Fig. 3(D), lane 6, a weak DNA-protein complex signal was observed under these conditions. These results indicate that Pc-ACE1, similar to Sc-ACE1 (Buchman et al., 1989), requires $\mathrm{Cu}^{+}$for binding to the DNA probe.

\section{PC-ACE1 mediates the activation of ACE- containing promoters}

Three different lines of evidence strongly suggest that PcACE1 mediates mcol promoter activation: (a) the copperdependent increase of mcol-mRNA; (b) the presence of two ACE elements in the promoter region of this gene and (c) the copper-dependent DNA-binding of Pc-ACE1 to one of these two ACE elements. In order to confirm that PcACE1 is involved in the copper-dependent transcription induction of the mcol promoter, a cell-free transcription system was employed. This consisted of a linearized plasmid containing the mcol promoter region linked to an EGFP reporter gene, a HeLa nuclear extract providing RNA polymerase plus general transcription factors and the Pc-ACE1 protein (see Methods). The basal level of transcription was greatly increased by adding Pc-ACE1 in the presence of $\mathrm{Cu}^{+}$(Fig. 4, lanes 1 and 2). In contrast, no transcriptional activation was observed when ascorbate was omitted, to prevent reduction of $\mathrm{Cu}^{2+}$ to $\mathrm{Cu}^{+}$(Fig. 4, lane 3 ) or when $\mathrm{Cu}^{+}$was chelated with $\mathrm{KCN}$ (Fig. 4, lane 4). Finally, the transcriptional activity of the $m c o 2$ promoter was also tested. In this case, no transcriptional activation was observed (Fig. 4, lanes 5 and 6).

\section{DISCUSSION}

In this work, we report that copper increases mRNA levels of mcol and mco2, but not of mco4. Among these genes, only the promoter of mcol showed the presence of two copper-dependent cis-acting regulatory elements known as ACE. These had been originally identified in the promoter regions of the $S$. cerevisiae copper metallothioneins cup1 and crs5, as well as in the promoter region of the superoxide dismutase gene sod1 (Culotta et al., 1989, 1994; Gralla et al., 1991). This element is the recognition site for the ACE1 transcription factor, which activates the transcription of target genes in response to copper. Prior to the present work, we had reported the cloning and sequencing of $P c$-acel, the gene encoding an ACE1 transcription factor in $P$. chrysosporium. However, until 


\section{A}

\section{S. cerevisiae cup 1 promoter}

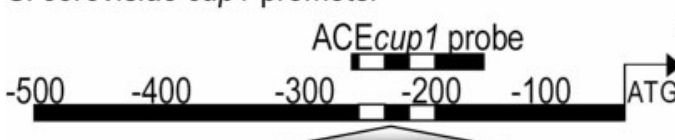

-219 5'-ATTCTTTTGCTGGCA-3' -234

-189 5'-TCTTTTTGGCTGAAC-3' -204

B

WT

5'-TCGCCACGACGAGCATGTACTTTGGCTGTAGGTCGCTCGTTTCGGT-3'

3'-GCTGCTCGTACATGAAACCGACATCCAGCGAGCAAAGCCAGCAGAT-5'

MUT
5'-TCGCCACGACGAGCATGTACETTGATGATAGGTCGCTCGTTTCGGT-3'
3'-GCTGCTCGTACATGCAACTACTATCCAGCGAGCAAAGCCAGCAGAT-5'

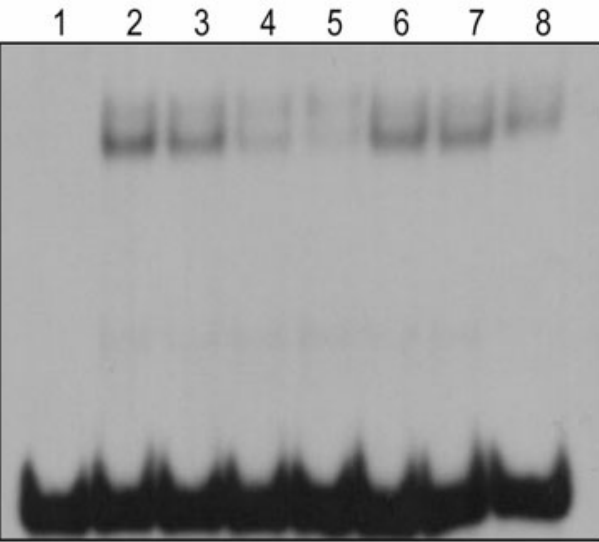

C

$\begin{array}{llllllll}1 & 2 & 3 & 4 & 5 & 6 & 7 & 8\end{array}$

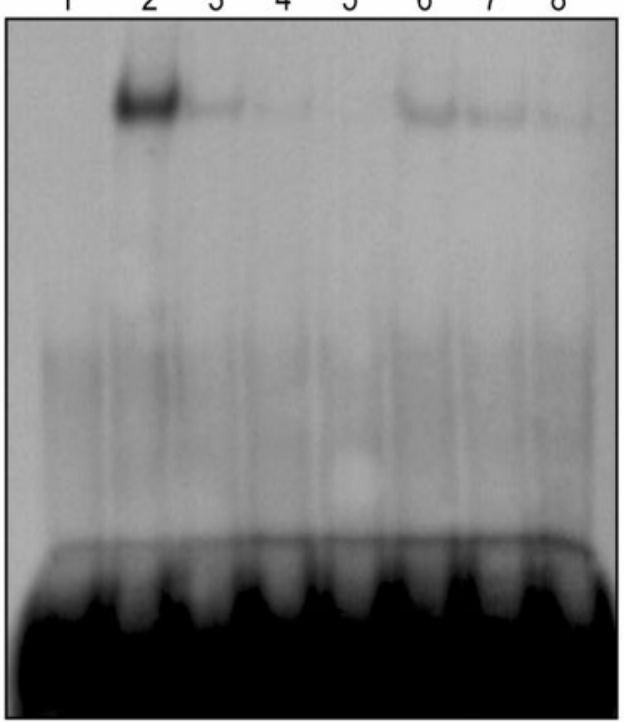

D

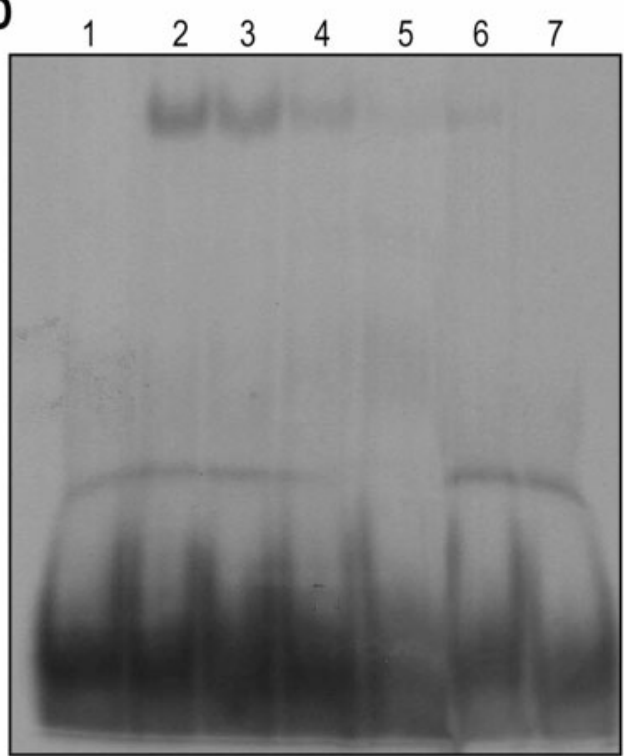

Fig. 3. Competition analysis by EMSAs. (A) Top, schematic representation of the S. cerevisiae cup1 gene promoter and its related 100 bp DNA probe containing two ACE elements (white boxes); the position of each element (superscript numbers) is indicated with respect to the translation start site (ATG). Bottom, binding assays conducted with this probe (ACEcup1) and in vitro-synthesized PC-ACE1 protein. Lanes contain: (1) free probe; (2) standard binding reaction without competitor; $(3,4,5)$ binding assays in the presence of 10-, 25- or 50-fold excess of unlabelled specific DNA probe, respectively; $(6,7,8)$ binding assays in the presence of 10-, 25- or 50-fold excess, respectively, of a 101 bp unrelated DNA as a non-specific competitor. (B) Synthetic oligonucleotides used as double-stranded competitors in the experiment shown in (C). Boxes denote the wild-type ACE element (WT) and the corresponding region within the mutant (MUT) competitor. Wild-type and substituted residues within the ACE consensus sequence are indicated in bold type. (C) Binding assays conducted with a 40 bp DNA probe containing the ACE element centred between positions -306 and -292 from the mco 1 translation start site (Fig. 2C) and the in vitro-synthesized Pc-ACE1 transcription factor. Lanes contain: (1) free probe; (2) standard binding reaction without competitor; $(3,4,5)$ binding assays in the presence of 10-, 25- or 50-fold excess unlabelled specific WT DNA probe, respectively; $(6,7,8)$ binding assays in the presence of 10-, 25- or 50-fold excess unlabelled MUT competitor, respectively. (D) Binding assays conducted with the same DNA probe and Pc-ACE1 transcription factor. Lanes contain: (1) free probe; (2) standard binding reaction; $(3,4,5)$ binding reactions in the presence of 25,50 or $100 \mathrm{mM} \mathrm{KCN}$, respectively, as $\mathrm{Cu}^{+}$-specific chelator; (6) binding reaction in the presence of $150 \mu \mathrm{M} \mathrm{Cu}^{2+}$ or (7) in the absence of copper. 


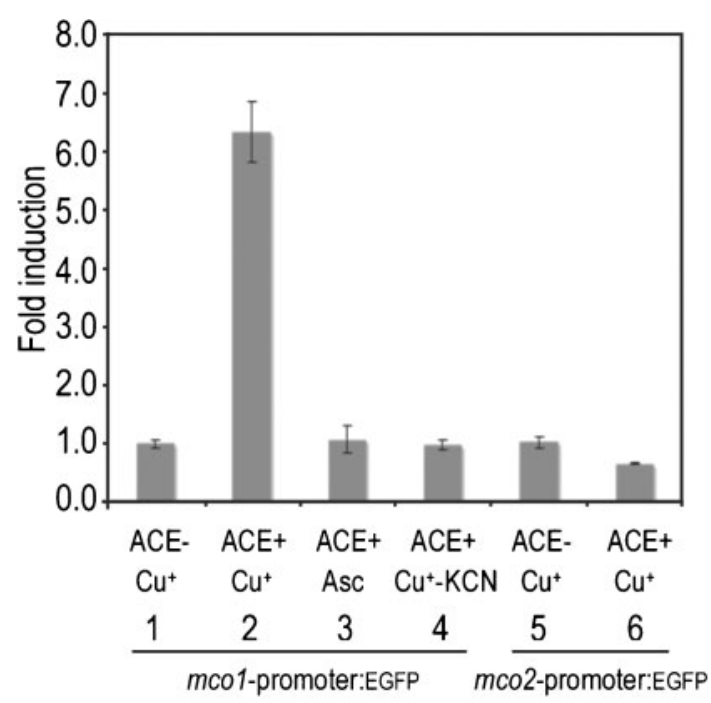

Fig. 4. PC-ACE1-dependent promoter activation of an ACEcontaining promoter. In vitro transcription of constructions mco1promoter: EGFP (lanes 1 to 4) and mco2-promoter : EGFP (lanes 5 and 6) as template DNAs. Reaction mixtures were supplemented with $\mathrm{Cu}^{+}$(added as $25 \mu \mathrm{M} \mathrm{CuSO}_{4}$-ascorbate, see Methods) and $3 \mu \mathrm{l}$ of either control rabbit reticulocyte lysate translation mixture (ACE-) or an identical mixture containing the in vitro-translated Pc-ACE1 transcription factor (ACE +). As a control, a reaction supplemented with $25 \mu \mathrm{M}$ ascorbate (Asc, lane 3 ) was carried out. $\mathrm{KCN}$, a Cu${ }^{+}$specific chelator, was also used $\left(\mathrm{Cu}^{+}-\mathrm{KCN}\right.$, lane 4). Transcripts were quantified by qRT-PCR $(n=3)$. Values are referred to the in vitro transcription assay carried out in the absence of Pc-ACE1 (lane 1).

now, the possible target gene(s) of Pc-ACE1 remained unknown.

The Pc-ace1 cDNA complements in vivo a mutant yeast strain lacking a functional ACE1 transcription factor. In this ACE1 $\Delta$ yeast strain, Pc-ACE1 was strictly required for growth in the presence of a high concentration of copper. Notably, Pc-ACE1 restored the copper inducibility of the yeast cupl gene (Polanco et al., 2006). In order to characterize the DNA-binding activity of Pc-ACE1, EMSAs were carried out using the in vitro-synthesized Pc-ACE1 transcription factor. This protein was tested with DNA probes containing ACE elements either from yeast or from $P$. chrysosporium. As a positive control, we also synthesized the $S$. cerevisiae ACE1 (Sc-ACE1). EMSAs using a DNA probe containing the two previously described ACE elements from the promoter region of the cup 1 gene from yeast (Culotta et al., 1989) showed specific DNA-protein interaction of both Sc-ACE1 (Fig. S1) and Pc-ACE1 (Fig. 3).

With each transcription factor, two DNA-protein complexes were obtained. Cysteine amino acids located within the copper-fist DNA-binding domain of Sc-ACE1 have been described as key residues that participate in DNAbinding activity and also as being involved in DNA target site specificity (Buchman et al., 1990). Since these cysteine residues are conserved in Pc-ACE1 (Polanco et al., 2006), a similar binding pattern of the two transcription factors was not unexpected. Two DNA-protein complexes had also been observed with Sc-ACE1 and a similar DNA probe (Hu et al., 1990). In that work, the authors observed that the lower-mobility complex was obtained only at high protein concentrations, whereas the higher-mobility complex was observed at both low and high protein concentrations. When the cup1 ACE elements are placed in separate probes, Sc-ACE1 binds efficiently, forming only one DNA-protein complex ( $\mathrm{Hu}$ et al., 1990). Consequently, it has been proposed that when both elements are located within the same DNA molecule, the higher-mobility complex corresponds to the occupancy of one DNA-binding site, whereas the DNA-protein complex showing a lower migration in the gel results from the occupancy of both ACE elements (Hu et al., 1990). This result clearly indicates that PcACE1, when interacting with and recognizing canonical ACE elements, behaves like Sc-ACE1. It also explains, at a molecular level, the reported copper-dependent Pc-ACE1mediated induction of the yeast cup1 gene in a ACE1 $\Delta$ background (Polanco et al., 2006).

The presence of two ACE elements in the promoter of mcol led us to examine the binding of Pc-ACE1 to at least one of them. The chosen element was centred between positions -306 and -292 from the mcol translation start site, similar to one of the ACE elements of the yeast cup1 gene (see Fig. 3). Fig. 3(C) illustrates that Pc-ACE1 does interact with this ACE element. The specificity of this interaction was tested in competition experiments with the same unlabelled probe and with an unlabelled probe including five point mutations. Four of these five mutations were located within the invariant 5'-GCTG-3' ACE core (Fig. 3B) (Gralla et al., 1991). Although some displacement of the complex was observed with the mutant probe, the complex was completely displaced with a 50 -fold molar excess of the unlabelled probe possessing the wild-type ACE element. This result indicates that the complex was formed by sequence-specific interactions, as has also been demonstrated for Cuf1, a Schizosaccharomyces pombe transcription factor that has a DNA sequence binding specificity similar to the ACE1 protein from Saccharomyces cerevisiae (Beaudoin et al., 2003). The requirement for $\mathrm{Cu}^{+}$ in the incubation mixture is also indicative of the specificity of this binding (Fig. 3D). It has been described that this metal, when binding to the copper regulatory domain within Sc-ACE1, stabilizes a specific tertiary fold forming a tetracopper thiolate cluster (Winge, 1998).

In order to demonstrate that Pc-ACE1 can stimulate the transcriptional activity of an ACE-containing promoter, an in vitro transcription assay was developed. As shown in Fig. 4, Pc-ACE1 enhances the transcription of a reporter gene linked to a DNA fragment encompassing $500 \mathrm{bp}$ upstream of the mcol translation start site. This promoter fragment contains the same ACE element first studied by EMSA. Interestingly, no transcriptional activation was observed in the absence of $\mathrm{Cu}^{+}$, or when $\mathrm{Cu}^{+}$was 
chelated with KCN. These results may account for the higher mcol-mRNA levels observed in vivo in cultures of $P$. chrysosporium supplemented with copper.

We also observed higher mco2-mRNA levels when cultures of $P$. chrysosporium were supplemented with copper. The absence of ACE elements in the promoter of mco2 made this result difficult to interpret. We then decided to test whether Pc-ACE1 can stimulate the transcription of a reporter gene linked to the promoter of $m c o 2$. As described in Results, this stimulation does not take place, suggesting that mco2 may not be a direct target of Pc-ACE1. In this regard, through hierarchical clustering of microarray data, a group of six differentially expressed genes that are highly induced in response to copper have been observed in $S$. cerevisiae. Among these, only cup1 and crs5, but not the other four genes, contain ACE elements in their respective promoter regions (van Bakel et al., 2005). It is conceivable that copper may induce gene expression by a mechanism different to that mediated by ACE1 transcription factor, such as, for example, the generation of reactive oxygen species. Clearly, further work is necessary to clarify the molecular mechanisms underlying the increment in mco2mRNA levels in response to copper.

\section{ACKNOWLEDGEMENTS}

This work was financed by the Millenium Institute for Fundamental and Applied Biology and by grant 1070588 from FONDECYT-Chile. P. C. is a predoctoral fellow supported by CONICYT-Chile.

\section{REFERENCES}

Baldrian, P. (2006). Fungal laccases - occurrence and properties. FEMS Microbiol Rev 30, 215-242.

Beaudoin, J., Mercier, A., Langlois, R. \& Labbé, S. (2003). The Schizosaccharomyces pombe Cufl is composed of functional modules from two distinct classes of copper metalloregulatory transcription factors. J Biol Chem 278, 14565-14577.

Breslauer, K. J., Frank, R., Blocker, H. \& Marky, L. A. (1986). Predicting DNA duplex stability from the base sequence. Proc Natl Acad Sci U S A 83, 3746-3750.

Brown, J. A., Li, D., Alic, M. \& Gold, M. H. (1993). Heat shock induction of manganese peroxidase gene transcription in Phanerochaete chrysosporium. Appl Environ Microbiol 59, 4295-4299.

Buchman, C., Scroch, P., Welch, J., Fogel, S. \& Karin, M. (1989). The CUP2 gene product, regulator of yeast metallothionein expression, is a copper-activated DNA-binding protein. Mol Cell Biol 9, 4091-4095.

Buchman, C., Skroch, P., Dixon, W., Tullius, T. D. \& Karin, M. (1990). A single amino acid change in CUP2 alters its mode of DNA binding. Mol Cell Biol 10, 4778-4787.

Carri, M. T., Galiazzo, F., Ciriolo, M. R. \& Rotilio, G. (1991). Evidence for co-regulation of $\mathrm{Cu}, \mathrm{Zn}$ superoxide dismutase and metallothionein gene expression in yeast through transcriptional control by copper via the ACE1 factor. FEBS Lett 278, 263-266.

Collins, P. J. \& Dobson, A. D. W. (1997). Regulation of laccase gene transcription. Appl Environ Microbiol 63, 3444-3450.

Culotta, V. C., Hsu, T., Hu, S., Fürst, P. \& Hamer, D. (1989). Copper and ACE1 regulatory protein reversibly induce yeast metallothionein gene transcription in a mouse extract. Proc Natl Acad Sci U S A 86, 8377-8381.

Culotta, V. C., Howard, W. R. \& Liu, X. F. (1994). CRS5 encodes a metallothionein-like protein in Saccharomyces cerevisiae. J Biol Chem 269, 25295-25302.

Evans, C. F., Engelke, D. R. \& Thiele, D. J. (1990). ACE1 transcription factor produced in $E$. coli binds multiple regions within yeast metallothionein upstream activation sequences. Mol Cell Biol 10, 426-429.

Galhaup, C., Goller, S., Peterbauer, C. K., Strauss, J. \& Haltrich, D. (2002). Characterization of the major laccase isoenzyme from Trametes pubescens and regulation of its synthesis by metal ions. Microbiology 148, 2159-2169.

Gralla, E. B., Thiele, D. J., Silar, P. \& Valentine, J. S. (1991). ACE1, a copper-dependent transcription factor, activates expression of the yeast copper zinc superoxide dismutase gene. Proc Natl Acad Sci U S A 88, 8558-8562.

Gross, C., Kelleher, M., lyer, V. R., Brown, P. O. \& Winge, D. R. (2000). Identification of the copper regulon in Saccharomyces cerevisiae by DNA microarrays. J Biol Chem 275, 32310-32316.

Hoegger, P. J., Kilaru, S., James, T. Y., Thacker, J. R. \& Kües, U. (2006). Phylogenetic comparison and classification of laccase and related multicopper oxidase protein sequences. FEBS J 273, 2308-2326.

Hu, S., Fürst, P. \& Hamer, D. (1990). The DNA and Cu binding functions of ACE1 are interdigitated within a single domain. New Biol 2, 544-555.

Karahanian, E., Corsini, G., Lobos, S. \& Vicuña, R. (1998). Structure and expression of a laccase gene from the ligninolytic basidiomycete Ceriporiopsis subvermispora. Biochim Biophys Acta 1443, 65-74.

Kersten, P. \& Cullen, D. (2007). Extracellular oxidative systems of the lignin-degrading basidiomycete Phanerochaete chrysosporium. Fungal Genet Biol 44, 77-87.

Larrondo, L. F., Salas, L., Melo, F., Vicuña, R. \& Cullen, D. (2003). A novel extracellular multicopper oxidase with ferroxidase activity in Phanerochaete chrysosporium. Appl Environ Microbiol 69, 6257-6263.

Larrondo, L. F., González, B., Cullen, D. \& Vicuña, R. (2004). Characterization of a multicopper oxidase gene cluster in Phanerochaete chrysosporium and evidence of altered splicing of the mco transcripts. Microbiology 150, 2775-2783.

Larrondo, L. F., Canessa, P., Melo, F., Polanco, R. \& Vicuna, R. (2007a). Cloning and characterization of the genes encoding the high affinity iron uptake protein complex Fet3/Ftr1 in the basidiomycete Phanerochaete chrysosporium. Microbiology 153, 1772-1780.

Larrondo, L. F., Canessa, P., Vicuna, R., Stewart, P., Vanden Wymelenberg, A. \& Cullen, D. (2007b). Structure and transcriptional impact of divergent repetitive elements inserted within Phanerochaete chrysosporium strain RP-78 genes. Mol Genet Genomics 277, 43-55.

Manubens, A., Avila, M., Canessa, P. \& Vicuña, R. (2003). Differential regulation of genes encoding manganese peroxidase $(\mathrm{MnP})$ in the basidiomycete Ceriporiopsis subvermispora. Curr Genet 43, 433-438.

Martin, F., Linden, T., Katschinski, D. M., Oehme, F., Flamme, I., Mukhopadhyay, C. K., Eckhardt, K., Tröger, J., Barth, S. \& other authors (2005). Copper-dependent activation of hypoxia-inducible factor (HIF)-1: implications for ceruloplasmin regulation. Blood 105, 4613-4619.

Martinez, A. T., Speranza, M., Ruiz-Dueñas, F. J., Ferreira, P., Camarero, S., Guillen, F., Martinez, M. J., Gutierrez, A. \& del Rio, J. C. (2005). Biodegradation of lignocellulosics: microbial, chemical, and enzymatic aspects of the fungal attack of lignin. Int Microbiol 8, 195-204. 
Palmieri, G., Giardina, P., Bianco, C., Fontanella, B. \& Sannia, G. (2000). Copper induction of laccase isozymes in the ligninolytic fungus Pleurotus ostreatus. Appl Environ Microbiol 66, 920-924.

Polanco, R., Lobos, S. \& Vicuña, R. (2002). Binding of nuclear proteins to the promoter region of the laccase gene Cs-lcs1 from the basidiomycete Ceriporiopsis subvermispora. Enzyme Microb Technol 30, 525-528.

Polanco, R., Canessa, P., Rivas, A., Larrondo, L. F., Lobos, S. \& Vicuña, R. (2006). Cloning and functional characterization of the gene encoding the transcription factor Acel in the basidiomycete Phanerochaete chrysosporium. Biol Res 39, 641-648.

Sanmartin, M., Pateraki, I., Chatzopoulou, F. \& Kanellis, A. K. (2007). Differential expression of the ascorbate oxidase multigene family during fruit development and in response to stress. Planta 225 , 873-885.

Soden, D. M. \& Dobson, A. D. W. (2001). Differential regulation of laccase gene expression in Pleurotus sajor-caju. Microbiology 147, 1755-1763.
Soden, D. M. \& Dobson, A. D. W. (2003). The use of amplified flanking region-PCR in the isolation of laccase promoter sequences from the edible fungus Pleurotus sajor-caju. J Appl Microbiol 95, 553-562.

Solomon, E. I., Sundaram, U. M. \& Machonkin, T. E. (1996). Multicopper oxidases and oxygenases. Chem Rev 96, 2563-2606.

Thiele, D. J. (1988). ACE1 regulates expression of the Saccharomyces cerevisiae metallothionein gene. Mol Cell Biol 8, 2745-2752.

Thorvaldsen, J. L., Sewell, A. K., McGowen, C. L. \& Winge, D. R. (1993). Regulation of metallothionein genes by the ACE1 and AMT1 transcription factors. J Biol Chem 268, 12512-12518.

van Bakel, H., Strengman, E., Wijmenga, C. \& Holstege, F. C. P. (2005). Gene expression profiling and phenotype analyses of $S$. cerevisiae in response to changing copper reveals six genes with new roles in copper and iron metabolism. Physiol Genomics 22, 356-367.

Winge, D. R. (1998). Copper-regulatory domain involved in gene expression. Prog Nucleic Acid Res Mol Biol 58, 165-195.

Edited by: M. Tien 